\title{
Neurology and the COVID-19 emergency
}

\author{
Alfredo Berardelli ${ }^{1,2}$ (D) Vincenzo Silani ${ }^{1,3}$. Paolo Barone ${ }^{1,4}$ - Paolo Calabresi ${ }^{1,5}$ - Paolo Girlanda ${ }^{1,6}$. \\ Leonardo Lopiano $^{1,7} \cdot$ Luca Massacesi $^{1,8}$. Salvatore Monaco ${ }^{1,9} \cdot$ Marco Onofrj $^{1,10}$. Cristina Tassorelli ${ }^{1,11}$. \\ Gioacchino Tedeschi ${ }^{1,12}$
}

Published online: 19 May 2020

(C) Fondazione Società Italiana di Neurologia 2020

Following the COVID-19 outbreak, significant changes have been implemented on a national level in the organization of neurology units and associated stroke units. Regionallydesignated COVID-19 hospitals have implemented an aggressive policy to relocate as many beds as possible to COVID-19 patients. In order to do so, the preferred strategy has been to reduce the number of beds in neurology units, and in some cases several units have been consolidated into one. In other cases, particularly in the northern regions, entire neurology units have been closed and converted into COVID-19 departments entrusted to other medical specialties. Some stroke units have followed the same path and become "hubs" and "spokes" for acute neurological pathology. In other situations, especially when it was necessary to meet the need for paramedical staff, the number of beds in neurology/stroke wards were significantly reduced and the nurses transferred to other units as needed to manage the COVID-19 emergency.

Alfredo Berardelli

alfredo.berardelli@uniroma1.it

1 Italian College of Professors of Neurology, Rome, Italy

2 Department of Human Neurosciences and IRCCS Neuromed Institute, Sapienza University of Rome, Rome, Italy

3 Department of Neurology-Stroke Unit and Laboratory Neuroscience, Istituto Auxologico Italiano, IRCCS, Department of Pathophysiology and Transplantation "Dino Ferrari Center", University of Milan, Milan, Italy

4 Department of Medicine, Surgery and Dentistry, University of Salerno, Salerno, Italy

5 Department of Neuroscience, Università Cattolica Sacro Cuore, Rome, Italy
During the COVID-19 emergency phase in March and April 2020, only patients with acute neurological diseases, including cerebrovascular disease, epilepsy, and other acute pathologies, were admitted to neurology units of COVID-19 hospitals. For all other patients with nonacute central and peripheral nervous system diseases, hospitalization was limited. Overall, the number of hospitalized neurological patients has undergone a drastic reduction. Some neurological patients have been hospitalized in non-COVID-19 hospitals or in other non-neurological wards, while a significant proportion of patients were forced to postpone their request for hospitalization. In addition to the reduced availability of beds, the COVID19 emergency has also resulted in the suspension of neurological day hospitals, ambulatory care, complex outpatient activity, and outpatient clinics, as well as clinics dedicated to the diagnosis and treatment of specific categories of neurological patients (centers for epilepsy, neu-
6 Department of Clinical and Experimental Medicine, University of Messina, Messina, Italy

7 Department of Neuroscience, University of Torino, Turin, Italy

8 Department of Neurosciences, Drug and Child Health, University of Florence, Florence, Italy

9 Department of Neurosciences, Biomedicine and Movement Sciences, University of Verona, Verona, Italy

10 Department of Neuroscience, Imaging and Clinical Sciences "G. D'Annunzio", University of Chieti, Chieti, Italy

11 Department of Brain and Behavioural Sciences, University of Pavia, IRCCS Mondino Foundation, Pavia, Italy

12 Department of Advanced Medical and Surgical Sciences, University of Campania "Luigi Vanvitelli”, Napoli, Italy 
romuscular diseases, movement disorders, dementia, headache, multiple sclerosis, amyotrophic lateral sclerosis, etc.).

In recent weeks, a slow but gradual reduction in the COVID-19 emergency has been observed, and consequently discussions are now taking place regarding the possible scaling down of the government's restrictive measures across the country.

Considering the importance and impact of neurological diseases on human health, it appears necessary to reopen, albeit gradually, all neurological hospital admissions throughout the country. Epidemiological data have overwhelmingly demonstrated the impact of neurological diseases on human health. Indeed, neurological diseases are among the leading causes of mortality (i.e. cerebral stroke) and many determine severe cognitive, mental, motor, and communication disabilities that may seriously limit the social functions of patients (neurodegenerative diseases) [1,2]. A further aspect that should not be underestimated involves possible postCOVID-19 sequelae, which may require accurate follow-up over time, as previously suggested by encephalitis lethargica [3] and postpolio syndrome [4].

Neurology therefore has an urgent need to ensure an adequate number of dedicated beds in university and nonuniversity hospitals in order to provide necessary care to a large number of patients. Besides acute neurological pathologies, neurological diseases include neurodegenerative conditions, complex diseases from a diagnostic and therapeutic point of view, and rare diseases that require specific experience and skills. All these conditions have a significant impact on society and families, who bear most of the burden in terms of care for these patients. For patients suffering from chronic neurological diseases, it is necessary to establish outpatient and dedicated care pathways where patients can be followed up periodically and where the effectiveness of therapeutic treatments in progress can be verified and monitored. The clinical course of these patients must also be able to accommodate scheduled admissions to neurology wards in the event of specific medical emergencies. Home healthcare also requires careful planning.

The COVID-19 emergency has resulted in the total suspension of all healthcare assistance and therapeutic pathways, which must be reopened and restored as soon as possible. We need to prepare ourselves for the future and for other emergencies by putting into practice the use of digital technologies that allow remote clinical evaluation and follow-up of patients. The application of new technologies, particularly those that permit the evaluation of patients at home, is already urgently needed in order to reduce the influx of patients to clinics and referral centers so as to comply with social distancing requirements.

COVID-19 reflects an unprecedented situation with a significant impact on the traditional organization of neurology on a national level. The awareness gained from this experience must propel us to carefully and proactively plan for the future so that the crucial role of neurology in managing emergencies and chronic degenerative diseases is safeguarded and optimized. The educational and proactive role of the academic component of the neurology field must serve as a point of reference, together with international and European regulations, in order to develop an adequate strategy for the future that takes into account the needs of the community, strategic changes, the contribution of new technologies, and the advancement of science.

\section{Compliance with ethical standards}

Conflict of interest None of the authors have any conflict of interest.

Ethical Approval The study was approved by the Ethical Committee.

\section{References}

1. Carroll WM (2019) The global burden of neurological disorders. Lancet Neurol 18:418-419

2. Feigin VL, Vos T, Nichols E, Carroll WM, Dichgans DG, Parmar P, Brainin M, Murray C (2020) The global burden of neurological disorders: translating evidence into policy. Lancet Neurol 19:255265

3. Lutters B, Foley P, Koehler PJ (2018) The centennial lesson of encephalitis lethargica. Neurology 90:563-567

4. Baj A, Colombo M, Headley JL, McFarlane JR, Liethof M-A, Toniolo A (2015) Post-poliomyelitis syndrome as a possible viral disease. Int J Infect Dis 35:107-116

Publisher's note Springer Nature remains neutral with regard to jurisdictional claims in published maps and institutional affiliations. 